\title{
A project to measure quantum spin correlations of relativistic electron pairs in Møller scattering
}

\author{
Jacek Ciborowski ${ }^{1, a}$, Paweł Caban ${ }^{2}$, Michał Drągowski $^{1}$, Joachim Enders ${ }^{3}$, Yuliya Fritzsche ${ }^{3}$, \\ Artem Poliszczuk ${ }^{1}$, Jakub Rembieliński ${ }^{2}$, and Marta Włodarczyk ${ }^{1}$ \\ ${ }^{1}$ University of Warsaw, Faculty of Physics, Pasteura 5, $02-093$ Warsaw, Poland \\ ${ }^{2}$ University of Łódź, Faculty of Physics and Applied Informatics, Pomorska 149/153, 90-236 Łódź, Poland \\ ${ }^{3}$ Technische Universität Darmstadt, Institut für Kernphysik, Schlossgartenstraße 2, 64289 Darmstadt, Ger- \\ many
}

\begin{abstract}
A project to measure quantum spin correlations for relativistic particles with mass is presented. It aims at testing spin observables in relativistic quantum mechanics. The measurement will be performed for pairs of entangled electrons originating from Møller scattering. A double Mott polarimeter, allowing for simultaneous measurement of the spin projections of both electrons, was designed for this purpose.
\end{abstract}

\section{Introduction}

\subsection{Review of spin correlations}

At the basis of the modern quantum mechanics and the quantum information theory lies the famous paper of Einstein, Podolsky and Rosen (EPR) [1] published in 1935. In this paper the completeness of quantum mechanics was put to doubt from the point of view of the local realism. The operational criterion of the local realism was given in 1964 by J. S. Bell [2]. It has a form of inequalities [2, 3] which should be fulfilled for every local realistic theory. The Bell-type inequalities involve the correlation functions, defined as: $C(A, B)=\sum_{\alpha \beta} \alpha \beta P_{\alpha \beta}$, where $\alpha$ and $\beta$ are the possible outcomes of measurements of some arbitrary observables $A$ and $B$, performed by two distant observers, Alice and Bob, respectively, and $P_{\alpha \beta}$ are the corresponding joint probabilities. The most common choices for $A$ and $B$ are the spin projections along given directions or polarisations. It has been shown experimentally that real quantum systems violate the Bell-type inequalities, both for photons [4] and massive particles [5].

A large majority of correlation experiments was performed with use of photons or nonrelativistic particles with mass. Since the pioneering paper by Czachor [6] one can notice a raise of interest in relativistic aspects of the quantum information theory, expressed in the most important papers on that subject [6,9-13], including the papers of our group [7, 8, 14-19]. However, the theoretical analysis of spin correlations in relativistic regime given in these papers is full of ambiguities and interpretational difficulties. They arise mostly due to the fact of an apparent conflict between nonlocality of the relativistic quantum mechanics and the Lorentz symmetry. Rembieliński et al. exhaustively discussed the

ae-mail: cib@fuw.edu.pl 
relativistic EPR correlation function for a bipartite system of spin-1/2 fermions in the framework of the relativistic quantum mechanics [17] and the quantum field theory [18]. The function was calculated in both pseudoscalar and vector state, which are the relativistic counterparts of the non-relativistic singlet and triplet states, respectively. The further analysis of the relativistic correlation functions for the first time showed their unexpected behaviour [7, 8]. Namely, for some fixed configurations it was found that the correlation functions are non-monotonic functions of particle momenta and may have local extrema. The degree of the violation of the Bell-type inequalities also depends on the particle momenta and in some cases is a non-monotonic function of the momentum. The correlation functions and the degree of violation of the Bell-type inequalities are different for different choices of the relativistic spin projection operator, but their qualitative behaviour is similar. The observed effect was also found for many other systems. An analysis of spin correlations in bipartite systems of spin-1/2 and spin-1 particles was performed [20]. The correlation functions were calculated for states which are not Lorentz scalars, for two different choices of relativistic spin projection operators. Again, in both cases there existed configurations for which the functions had local extrema. Local extrema were found in the correlation function in a hybrid system of a photon and a spin- $1 / 2$ fermion [21]. Correlations of relativistic vector bosons assuming that their linear polarisations (determined by helicity, a quantity which in contrast to spin is uniquely defined in relativistic quantum mechanics) is measured, were discussed by Caban [14]. The results confirm the earlier observation that the correlation function can, for some fixed configurations, have local extrema. Moreover, it was shown that for certain configurations the degree of the inequality violation increases with the particle momenta, reaching its maximal value in the ultrarelativistic limit, in contrast to suggestions of some authors [6, 9, 22]. All the above mentioned results strongly suggest that the existence of the local extrema, independent of the choice of the relativistic spin projection operator, is an immanent feature of relativistic correlations. It is also clear that the behaviour of the relativistic correlation functions may be different than in the non-relativistic case.

As far as we know, up to date there have been only three experiments testing Bell-type inequalities by means of massive relativistic spin one-half particles: the Lamehi-Rachti-Mittig (LRM) experiment [23] performed about thirty years ago in CEN-Saclay and two recent experiments: the first one given at the Kernfysisch Versneller Instituut (KVI, Holland) by S. Hamieh et al. [24] and the second one performed by H. Sakai et al. [25] in RIKEN Accelerator Research Facility (Japan). In all three experiments the proton-proton spin correlations were measured. LRM team tested Bell-type inequalities with use of the low energy $(13.5 \mathrm{MeV})$ proton beam which corresponds to the proton velocity $v \sim 0.17 c$. For the other hand, in KVI experiment, the spin correlations of proton pairs in a ${ }^{1} S_{0}$ intermediate state, obtained from ${ }^{12} \mathrm{C}\left(d,{ }^{2} \mathrm{He}\right){ }^{12} \mathrm{~B}$ nuclear charge-exchange reaction, were measured for protons with the kinetic energy $\sim 86 \mathrm{MeV}(v \sim 0.4 c)$. Finally, in the RIKEN experiment the proton pair was created in the ${ }^{1} \mathrm{H}\left(d,{ }^{2} \mathrm{He}\right) n$ charge-exchange reaction with the proton energy $\sim 135 \mathrm{MeV}$ $(v \sim 0.5 c)$. All these experiments were in agreement with the non-relativistic quantum mechanics predictions. The particles were too slow to give a significant difference between predictions of relativistic and non-relativistic quantum mechanics. From our estimation it follows that in a conclusive experiment the kinetic energy of the particles used should be at least of order of the particles rest mass.

In view of the above, we have undertaken a task of measuring the Einstein-Podolsky-Rosen (EPR) spin correlations for electron pairs originating from the Møller scattering in the relativistic regime, which is already achieved for MeV-energy electrons. This is the first project addressing the issue of quantum spin correlations of relativistic massive fermions. 


\subsection{Spin correlations in Møller scattering - theory}

Given two correlated spin-1/2 fermions, one can measure their spin projections separately on given directions and the results are either $+1 / 2$ or $-1 / 2$ for each of them. Let $P_{s 1 s 2}$, denote the probability that the fermions had spin projections $s 1$ and $s 2$, respectively, where $s 1$ and $s 2$ are either + or (along the positive or negative direction of the axis). The correlation function can then be expressed as: $C=P_{++}+P_{--}-P_{-+}-P_{+-}$. The probabilities $P_{s 1 s 2}$ are the primary subject of our measurement using the method of Mott polarimetry (below). Calculations regarding the correlation function and the corresponding probabilities, in the context of the present project, have already been completed [26] and also reported at this Conference [27]. As an example, the dependence of $P_{++}, P_{--}, P_{-+}$and $P_{+-}$ on beam energy is shown in fig. 1. The correlation function and the probabilities depend on the beam energy and polarisation as well as on the final state electron configurations, including a dependence on the directions along which spin projections are measured, $\vec{a}$ and $\vec{b}$.

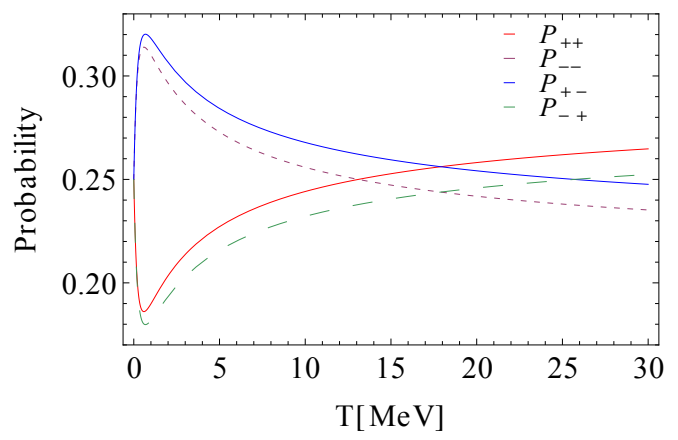

Figure 1. Dependence of the probabilities on beam energy ( $3 \mathrm{MeV}$ chosen for this measurement). Plot made for a symmetric scattering and vectors $\vec{a}$ and $\vec{b}$ in the scattering plane.

\section{Experiment}

\subsection{Preliminaries}

We are planning to carry out the measurement using a $3 \mathrm{MeV}$ polarised electron beam available at the S-DALINAC accelerator in the Institut für Kernphysik, Technische Universität Darmstadt. Beam electrons will scatter on atomic electrons in a $25 \mu \mathrm{m}$-thick Be target (as well as on Be nuclei which constitutes the main source of background). This particular choice of beam energy allows to collect sufficient statistics while the Møller electrons are still highly relativistic, approx. $1.5 \mathrm{MeV}$ each, in a symmetric scattering configuration, i.e. at $\theta=16.75^{\circ}$. The corresponding differential cross section for this process amounts to approx. $100 \mathrm{mb} / \mathrm{sr}$. Longitudinal, transverse and intermediate beam polarisations are available in the range of values from 0.35 to 0.85 .

\subsection{Mott scattering}

Spin projections on predefined directions will be measured by means of the Mott polarimetry. The differential cross section for scattering of a polarised electron off a nucleus is given by the following formula:

$$
\frac{\mathrm{d} \sigma}{\mathrm{d} \Omega}=\left(\frac{\mathrm{d} \sigma}{\mathrm{d} \Omega}\right)_{0}[1+S(\theta) \vec{P} \vec{n}]
$$


where $(\mathrm{d} \sigma / \mathrm{d} \Omega)_{0}$ corresponds to the scattering of unpolarised electrons, $S(\theta)$ is the Sherman function (depending on the electron scattering angle, $\theta$ ) and $\vec{P} \vec{n}$ is the electron spin projection on a direction given by a unit vector $\vec{n}$, perpendicular to the scattering plane. The Sherman function, calculated for a single nucleus (zero-thickness target), in terms of the electron energy and scattering angle (contour plot), is shown in fig. 2. The higher value of the Sherman function, the higher sensitivity of scattering

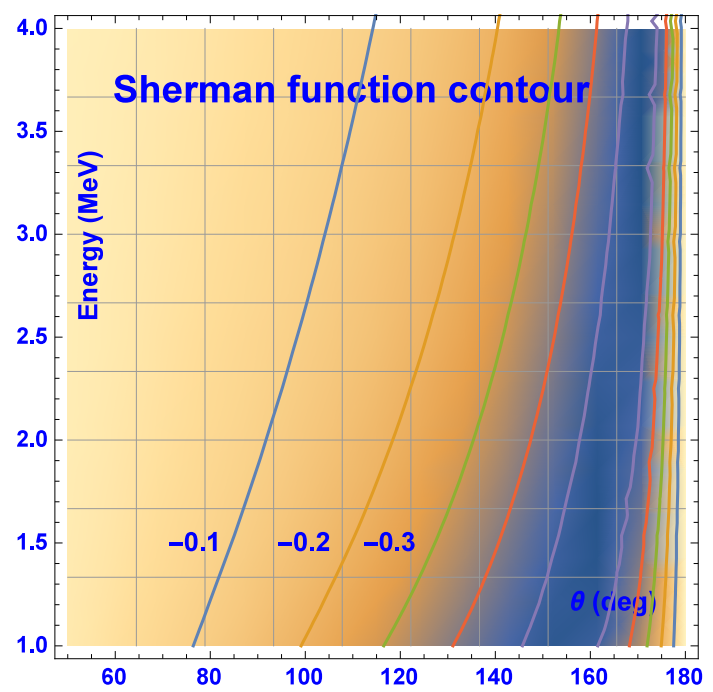

Figure 2. Sherman function contour: energy $(\mathrm{MeV})$ vs. scattering angle $\theta$ (degrees), in the MeV-energy range.

to the electron polarisation. For the Møller electrons of about $1.5 \mathrm{MeV}$ energy, the lowest acceptable value of $S \approx-0.2$ implies scattering at about $120^{\circ}$. In practice, Mott targets must have finite thickesses, $d$, and in consequence the corresponding effective Sherman function take smaller values. The effective Sherman function can be approximately parametrised as: $S(d)=S_{0} /(1+\alpha d)$, where $S_{0}$ is the function for a single nucleus (zero-thickness target) and $\alpha$ - a parameter (the so called dilution factor). The optimal target thickness equals $1 / \alpha$ for this parametrisation and can be derived by maximising the figure-of-merit, given by: $S^{2}(d) d$. The effective Sherman function drops to a half of its theoretical value, $S_{0}$, for a target of the optimal thickness. Since the procedure of deriving probabilities $P_{s 1 s 2}$ requires knowledge of the effective Sherman function in the conditions of the experiment, it has to be measured or at least reliably simulated, which is also a part of dedicated activities in this project [28].

\subsection{Double Mott polarimetry}

The idea of measuring spin correlations of electron pairs described in this article, boils down to measuring four numbers, as described below. The sequence of Møller - Mott scatterings is illustrated on fig. 3 which depicts forward Mott scatterings for clarity while the real measurement will be done for backward scattering (angles about $120^{\circ}$ ). Assume two hypothetical planes, each perpendicular to the respective Møller electron and a ring drawn at each plane with the centre at the incident electron track. Two oppositely located areas (ring sections) are marked black at each ring, corresponding to scattering to the left or to the right off the centre, marked by letters $\mathrm{L}$ and $\mathrm{R}$ for each of the two rings. Events of 
interest are those in which each electron hits either the respective area $\mathrm{L}$ or $\mathrm{R}$. This way four coincidences can be defined and the corresponding numbers of events counted, marked: $L_{1} L_{2}, L_{1} R_{2}, R_{1} L_{2}$ and $R_{1} R_{2}$. The centres of the two areas and the entry point at the Mott target for each Møller electron define a plane to which the perpendicular direction is that at which the spin projection of each electron is measured. These directions are marked by vectors $\vec{a}$ and $\vec{b}$, corresponding to $\vec{n}$ in (1).

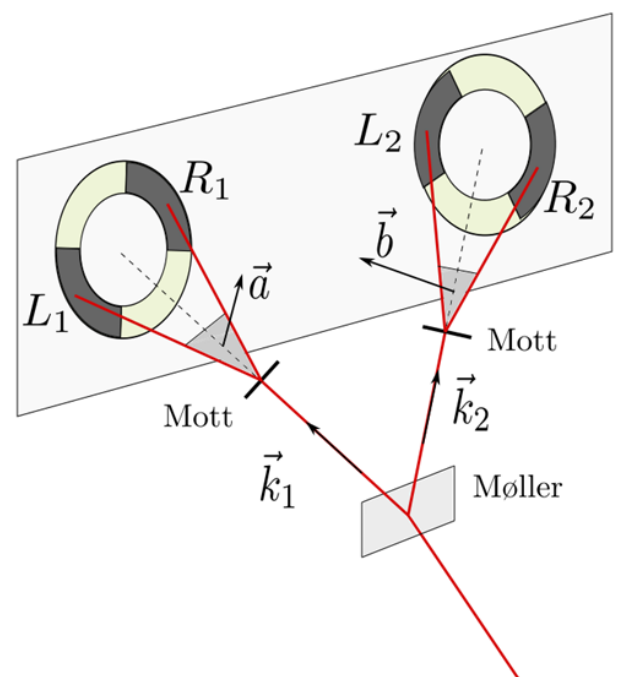

Figure 3. Illustration of the method of measuring coincidences $L_{1} L_{2}, L_{1} R_{2}, R_{1} L_{2}$ and $R_{1} R_{2}$, needed for deriving probabilities of spin projections, $P_{++}, P_{--}, P_{-+}, P_{+-}$(see text).

\subsection{Derivation of results}

The probabilities of spin projections $P_{++}, P_{--}, P_{-+}, P_{+-}$can be determined from four numbers of coincidences $L_{1} L_{2}$ etc. as follows. First the probabilities of the above coincidences are defined, $P_{L L}$, $P_{R R}, P_{L R}$ and $P_{R L}$, by normalising the numbers to the total. Then, following Sakai et al. [25], one can compute the probabilities from the following formula:

$$
\left(\begin{array}{l}
P_{++} \\
P_{--} \\
P_{+-} \\
P_{-+}
\end{array}\right)=\frac{1}{\bar{S}_{1} \bar{S}_{2}}\left(\begin{array}{cccc}
c_{++} & c_{--} & -c_{+-} & -c_{-+} \\
c_{--} & c_{++} & -c_{-+} & -c_{+-} \\
-c_{+-} & -c_{-+} & c_{++} & c_{--} \\
-c_{-+} & -c_{+-} & c_{--} & c_{++}
\end{array}\right)\left(\begin{array}{c}
P_{L L} \\
P_{R R} \\
P_{L R} \\
P_{R L}
\end{array}\right),
$$

where $P_{L L}=N_{L L} / N_{0}$ etc., $N_{L L}, N_{R R}, N_{L R}$ and $N_{R L}$ denote the number of given coincidences and $N_{0}=N_{L L}+N_{R R}+N_{L R}+N_{R L}$. The coefficients $c_{i j}$ are defined as: $c_{++}=\left(1+\bar{S}_{1}\right)\left(1+\bar{S}_{2}\right) / 4$, $c_{--}=\left(1-\bar{S}_{1}\right)\left(1-\bar{S}_{2}\right) / 4$ etc., and $\bar{S}_{1}, \bar{S}_{2}$ are the effective values of the Sherman function for the Mott scattering into the selected ring section (fig. 3) for the first and the second Møller electron, respectively. It can be seen from (2) that precise knowledge of the Sherman function in terms of the electron scattering angle and energy (in the range of this measurement) is necessary for determining accurate values of probabilities, given the measured numbers of coincidences. 


\subsection{The double polarimeter}

The prototype of the double polarimeter is shown in fig. 4. It is built of the following parts:

- The main pipe, which will be a part of the accelerator vacuum system, attached with a flange (top left) and closed with a beam dump at the bottom right end; $3 \mathrm{MeV}$ electrons will enter from the side of a flange

- Two pipes (Møller legs) welded into the main pipe at $\theta=16.75^{\circ}$ which will select two electrons after a symmetric scattering of the beam on atomic electrons in a $20 \mu \mathrm{m}$ Be target, which will be placed at the crossing of the leg axes

- Two Mott polarimeters, each consisting of a pair of pipes (Mott arms) welded at $120^{\circ}$ into each Møller leg (backscattering). Each Mott arm will be equipped with a $10 \mu \mathrm{m}$ Au Mott target. The downstream part of the Møller leg will have a larger diameter to reduce backscattering background and will be closed with an Møller electron dump

- Each Mott arm will be equipped with a scintillation counter

- All above enumerated pipes will be equipped with collimators

- Directions for measuring spin projections, $\vec{a}$ and $\vec{b}$, can be selected by appropriate orientation of the arms of the Mott polarimeters.

The angular acceptance for a $3 \mathrm{MeV}$ beam is planned to be approx. $3 \times 10^{-4} \mathrm{sr}$ for the Møller electrons and $7 \times 10^{-3}$ sr for the Mott electrons. The event rate is expected to be approximately $10^{-16}$ per beam electron. The prototype is currently under construction and testing.

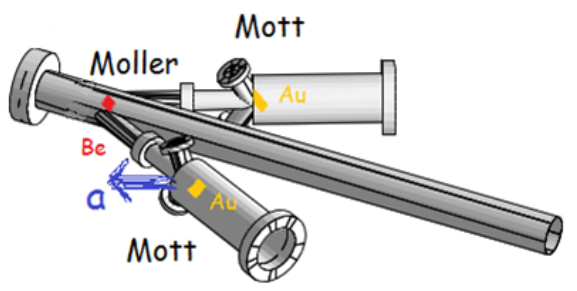

Figure 4. A view of the double polarimeter. The electron beam enters from the upper left corner and scatters on the Møller (Be) target. Two final state electrons enter the pipes at which two Mott polarimeters are installed. Each electron scatters off the Mott Au target in the respective polarimeter and is recorded in the scintillation counter, attached through a flange to each polarimeter arm (not shown). Selecting a relative orientation of the vectors $\vec{a}$ and $\vec{b}$ (in a limited range) is done by appropriate screwing of the polarimeter and the Møller pipe flanges (one of the vectors, $\vec{a}$, is marked on the figure).

Simulations of the response of the double polarimeter using the Geant4 [29] package is in progress. Electrons detected in scintillation counters are predominantly the beam electrons ( $3 \mathrm{MeV}$ ) and Møller electrons (about 1.5 MeV). A significant fraction of electrons emerging from the Be target loose some energy by undergoing scatterings on inner walls of the pipes. Since the polarimeter has no tracking, it is of key importance to have a correct modelling and understanding of its performance. Preliminary results also indicate a low-energy photon background entering the scintillators. It is expected that 
the requirement of a coincidence in two counters (as explained above) will eliminate most of this background as well as beam scattering events. The response of the scintillation counters is simulated in parallel using both Geant4, as well as the FLUKA [30] and PHOTRACK [31] packages, the latter dedicated to light collection in the scintillator-lightguide assembly.

\subsection{The beam}

The measurement is planned at the S-DALINAC electron accelerator, located in the Institut für Kernphysik of the Technische Univeristät Darmstadt, Germany. It offers a $3 \mathrm{MeV}$ polarised electron beam with longitudinal and transverse polarisation ranging from 0.35 to 0.85 . The beam intensity reaches approx. $10^{14}$ electrons/s which would yield of order $10^{2}$ useful events per day.

\section{Summary and conclusions}

In summary, the present project constitutes a first attempt to measure spin correlations of relativistic electron pairs (final state of the Møller scattering). The main aim of the measurement is to draw conclusions regarding spin observables in relativistic Quantum Mechanics. A prototype of a dedicated double Mott polarimeter is currently under construction in parallel to partial laboratory tests using a beta-electron source. Data taking, depending on the S-DALINAC availability, is forseen in 2018.

This work was partially supported from the funds of the National Science Centre, contract/decision DEC-2012/06/M/ST2/00430 and as a postdoc project (M.W.) contract/decision DEC2013/08/S/ST2/00551.

\section{References}

[1] A. Einstein, B. Podolsky, and N. Rosen, Phys. Rev. 47, 777 (1935)

[2] J. S. Bell, Physics, 1, 195 (1964)

[3] J. F. Clauser et al., Phys. Rev. Lett. 23, 880 (1969)

[4] A. Aspect, J. Dalibard, and G. Roger, Phys. Rev. Lett. 49, 1804 (1982)

[5] M. A. Rowe et al., Nature 409, 791 (2001)

[6] M. Czachor, Phys. Rev. A55, 72 (1997)

[7] P. Caban, J. Rembieliński and M. Włodarczyk, Phys. Rev. A77, 012103 (2008)

[8] P. Caban, J. Rembieliński and M. Włodarczyk, Phys. Rev. A79, 014102 (2009)

[9] H. Terashima and M. Ueda, Int. J. Quantum Information, 1, 93 (2003)

[10] A. Peres, P. F. Scudo and D. R. Terno, Phys. Rev. Lett. 88 230402, (2002)

[11] M. Czachor and M. Wilczewski, Phys. Rev. A68, 010302(R) (2003)

[12] A. Peres and D. R. Terno, Rev. Mod. Phys. 76, 93 (2004)

[13] P. M. Alsing and G. J. Milburn, Quantum Information and Computation, 2, 487 (2002)

[14] P. Caban, Phys. Rev. A77, 062101 (2008)

[15] P. Caban and J. Rembieliński, Phys. Rev. A59, 4187 (1999)

[16] J. Rembieliński and K. A. Smoliński, Phys. Rev. A66, 052114 (2002)

[17] P. Caban and J. Rembieliński, Phys. Rev. A72, 012103 (2005)

[18] P. Caban and J. Rembieliński, Phys. Rev. A74, 042103 (2006)

[19] P. Caban, Phys. Rev. A76, 052102 (2007)

[20] P. Caban, J. Rembieliński and M. Włodarczyk, Phys. Rev. A83, 034101 (2010) 
[21] P. Caban, J. Rembieliński, P. Witas and M. Włodarczyk, Phys. Rev. A83, 032115 (2011)

[22] D. Ahn et al., Phys. Rev. A67, 012103 (2003)

[23] M. Lamehi-Rachti and W. Mittig, Phys. Rev. D14, 2543 (1976)

[24] S. Hamieh et al., J. Phys. G: Nucl. Part. Phys. 30, 481 (2004)

[25] H. Sakai et al., Phys. Rev. Lett. 97, 150405 (2006)

[26] P. Caban, J. Rembieliński and M. Włodarczyk, Phys. Rev. A88, 032116 (2013)

[27] M. Wlodarczyk et al., this conference

[28] M. Drạgowski et al., accepted to Nucl. Inst. Meth. in Phys. Res. B (2016)

[29] S. Agostinelli et al., Nucl. Inst. Meth. in Phys. Res. A 506, 250 (2003)

[30] A. Ferrari, P.R. Sala, A. Fasso and J. Ranft, FLUKA: A multi-particle transport code (Program version 2005), No. INFN-TC-05-11 (2005)

[31] N. Ghal-Eh, M.C. Scott, R. Koohi-Fayegh and M.F. Rahimi, Nucl. Inst. Meth. in Phys. Res. A 516, 116 (2004) 